\title{
ACCELERATION OF LEAD IONS IN THE CERN PS BOOSTER AND THE CERN PS
}

F. Blas, P. Bossard, R. Cappi, G. Cyvoct, R. Garoby, G. Gelato, H. Haseroth, E. Jensen, D. Manglunki, K. Metzmacher, F. Pedersen, N. Rasmussen, K. Schindl, G.C. Schneider, H. Schönauer, L. Sermeus, M. Thivent, M. van Rooij, F. Völker, E. Wildner, CERN, CH-1211 Geneva 23, Switzerland

\section{INTRODUCTION, BASIC PARAMETERS}

The new CERN Heavy-Ion Accelerating Facility [1] implies besides a new linac also important modifications of existing accelerators. They are imposed by the low speed and the low intensity of the ion beam and, crucially at low energy, by the short lifetime of the partially stripped ions due to charge exchange with the atoms of the residual gas.

Once the optimum charge state $\left(\mathrm{Pb}^{53+}\right)$ and energy of the injector $(4.2 \mathrm{MeV} / \mathrm{u})$ had been chosen [2], the operational pulse-to-pulse variability (PPM) of particle species and intensities in the PS and its four-ring Booster (PSB) dictate the main beam parameters (Table I).

Table I: Basic parameters of lead ion and proton beams

\begin{tabular}{|l|c|c|}
\hline \multicolumn{1}{|c|}{ Parameter } & ${ }^{208} \mathbf{P b}^{\mathbf{5 3 +}}$ & $\mathbf{p}$ \\
\hline Injection PSB & $4.2 \mathrm{MeV} / \mathrm{u}$ & $50 \mathrm{MeV}$ \\
\hline Kinetic Energy & 0.0946 & 0.314 \\
\hline$\beta=\mathrm{v} / \mathrm{c}$ & $0.141 \mathrm{~T}$ & $0.125 \mathrm{~T}$ \\
\hline Bending Field B & $1.8 \mathrm{~T} / \mathrm{s}$ & $0.4 \mathrm{~T} / \mathrm{s}$ \\
dB/dt & 17 & 5 \\
\hline RF Harmonic Number &
\end{tabular}

PSB Intermediate flat porch

\begin{tabular}{|l|c|l|}
\hline Kinetic Energy & $32.4 \mathrm{MeV} / \mathrm{u}$ & \\
\hline$\beta=\mathrm{v} / \mathrm{c}$ & 0.257 & \\
\hline RF Harmonic Number & $17 / 10$ & \\
\hline
\end{tabular}

Ejection flat top

\begin{tabular}{|l|c|c|}
\hline Kinetic Energy & $95.4 \mathrm{MeV} / \mathrm{u}$ & $1 \mathrm{GeV}$ \\
\hline RF Harmonic Number & $10 / 5$ & 5 \\
\hline Acceleration Time & $288 \mathrm{~ms}$ & $415 \mathrm{~ms}$ \\
\hline PS Harmonic Number & 20 & 20 \\
\hline
\end{tabular}

PS Extraction

\begin{tabular}{|l|c|c|}
\hline Kinetic Energy & $4.25 \mathrm{GeV} / \mathrm{u}$ & $19.07 \mathrm{GeV}$ \\
\hline Acceleration Time & $0.5 \mathrm{~s}$ & $0.5 \mathrm{~s}$ \\
\hline
\end{tabular}

\section{PSB INJECTION LINE AND INJECTION}

The magnetic rigidity of the ion beam being $13 \%$ higher than the proton one, all elements of the injection line have to vary in PPM by this amount and keep their values over the $500 \mu$ s duration of the linac pulse. While most elements and their power supplies were able to do this with some minor upgrading, a dedicated pulsed staircase magnet (vertical distributor) had to be designed to switch the ion beam to the four Booster rings. The multi-turn injection kicker generators were upgraded to provide in PPM two different negative slopes. The steering of the ion beam through the line is monitored solely by scintillator screens, which had to be made operating in PPM to avoid interference with proton pulses.

\section{VACUUM LIMITED LIFETIME AND RELATED HARDWARE UPGRADES}

The partially stripped ions are subject to charge exchange processes with the residual gas. Empirical formulae derived from cross-section measurements [3] predicted unbearable loss at the existing PSB vacuum level of $210^{-8} \mathrm{mbar}$ and suggested necessary nitrogen equivalent pressures of a few $10^{-9} \mathrm{mbar}$, yielding calculated lifetimes (cf. Figure 1 in [2]) of $60 \mathrm{~ms}$ at injection and $1.5 \mathrm{~s}$ at extraction, with a steep slope at low energies. Therefore the - unbakeable - vacuum system had to be improved. The possible measures were limited to adding 14 ion getter pumps and $40 \mathrm{Ti}$ sublimation pumps in the PSB with the aim to lower the static pressure to $3.710^{-9} \mathrm{mbar}\left(510^{-9}\right.$ mbar achieved). In the PS some 90 Ti sublimation pumps reduced the pressure from $510^{-8}$ to less than $510^{-9}$ mbar. Further improvements, including more refined instrumentation, are under study [4].

Even with this vacuum pressure, fastest possible acceleration is mandatory for an acceptable transmission in the Booster. The vacuum is less critical in the PS as lifetimes at $410^{-9} \mathrm{mbar}$ are of the order of seconds. The PSB main power converter is now routinely operating with four rectifier groups instead of three to allow for higher induced voltages and $\mathrm{dB} / \mathrm{dt}$. Equally, the limitations of $\mathrm{d}^{2} \mathrm{~B} / \mathrm{dt}^{2}$ had to be raised in order to reach the $\mathrm{dB} / \mathrm{dt}$ limit faster. A further, decisive, shortening of the critical lowenergy phase is to inject and capture not on a flat bottom but at rather large $\mathrm{dB} / \mathrm{dt}$.

\section{PSB CAPTURE AND ACCELERATION CYCLE}

Three distinct loss mechanisms prevail during RF capture and initial acceleration (Figure 1): (i) initial fast loss due to transverse scraping of the spiralling beam before being captured, (ii) loss of about $10 \%\left(\tau_{\text {ini }}=15 \mathrm{~ms}\right)$ due to particles not captured due to the lack of adiabaticity 
$\left(\mathrm{k}_{\mathrm{a}}=2.4\right)$ [5], and (iii) after $4 \mathrm{~ms}$, slow loss $(\tau=39 \mathrm{~ms})$ due the vacuum limited lifetime at low energy. Given this lifetime and the constraints on $\mathrm{dB} / \mathrm{dt}$ and $\mathrm{d}^{2} \mathrm{~B} / \mathrm{dt}^{2}$ from the main power converter, an optimum value for $\mathrm{dB} / \mathrm{dt}$ at injection was found at $1.8 \mathrm{~T} / \mathrm{s}$.

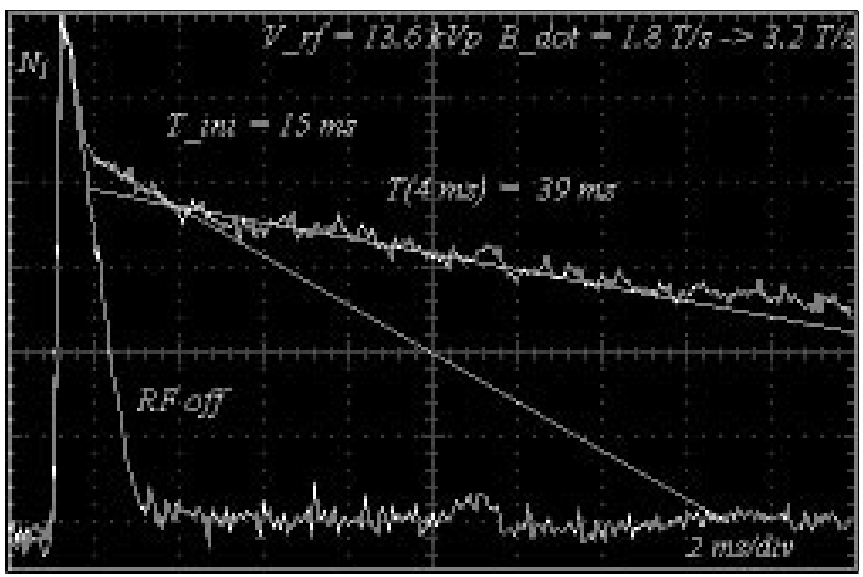

Figure 1: Beam current during horizontal multi-turn injection and capture in the PSB with and without RF.

Initial acceleration is with harmonic number 17 . When the $8.3 \mathrm{MHz}$ frequency limit is reached, an adiabatic debunching followed by an adiabatic rebunching with $h=10$ takes place at an intermediate flat top followed by further acceleration to maximum energy (Figure 2). A second harmonic number change from 10 to 5 at the ejection flat top serves to facilitate bucket to bucket transfer to the $h=20 \mathrm{RF}$ system of the PS.

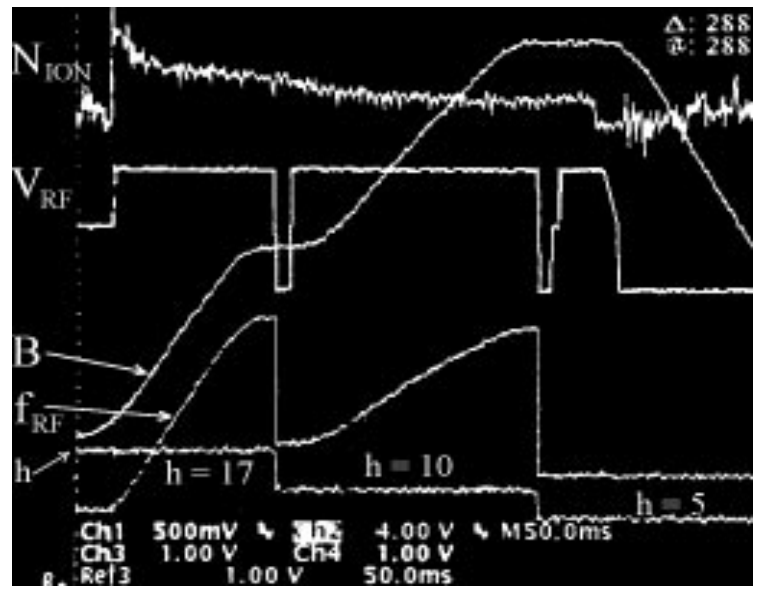

Figure 2: Acceleration in the PSB. From top to bottom: Ion intensity, RF voltage, main B field, RF frequency, harmonic number, vs. time

Four complete, new, digital beam control systems with digitally programmed acceleration frequency (smoothed by an AC-coupled beam phase loop) were built using the new standard modules recently developed for machines in the PS complex [6].

\section{TRANSFER TO PS AND PS INJECTION}

In order to eject and merge the slow ion beams from the four PSB rings sequentially into the PS, a total of $5 \mathrm{~km}$ of new, longer, high quality $\mathrm{SF}_{6}$-filled PFN cables were installed for the corresponding seven fast kicker pulse generators. The main pulse switches were replaced by more powerful thyratrons to avoid pulse quenching. At PS injection, the double flat-top pulse length is obtained by pulsing two groups of kicker modules sequentially. The magnets of each group are short-circuited (in PPM) to double their strengths.

\section{ACCELERATION IN THE PS}

To minimize the SPS filling time the PS cycle length was limited to $1.2 \mathrm{~s}$. This resulted in a maximum energy of $4.25 \mathrm{GeV} / \mathrm{u}(\gamma=5.56)$, just below transition $\left(\gamma_{\mathrm{t}}=6.12\right)$.

The beam control previously used for the acceleration of sulfur ions was upgraded to the new standard recently developed for machines in the PS complex [6]. After adjustment of RF capture and acceleration, about $25 \%$ of the beam was still lost by stripping on the residual gas (Figure 3). These losses increase up to $30-40 \%$ when the ion cycle is preceded by a lepton cycle due to outgassing because of synchrotron radiation impact.

The energy stability of the extracted beam proved to be insufficient with the frequency programme alone because of the proximity of transition. A rudimentary radial loop was then assembled and guaranteed a maximum peak deviation $\Delta \mathrm{p} / \mathrm{p}=110^{-4}$.

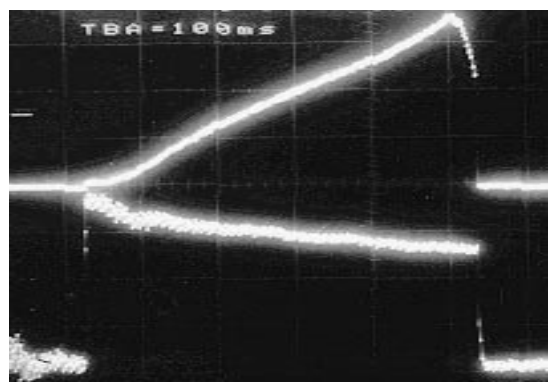

Figure 3: Acceleration in the PS (100 ms/div).

Peak (top trace) and DC (bottom trace ) beam current.

\section{FULL STRIPPING}

Total ion stripping, from $\mathrm{Pb}^{53+}$ to $\mathrm{Pb}^{82+}$, is performed with an Al foil situated in the PS-SPS transfer line. The foil is mounted on a mechanism which moves it into the beam only during the ion cycles. Three foil thicknesses were tested, and first preliminary results are shown in Table II. 
Table II: Relative momentum loss, approximate stripping efficiency, measured and theoretical transverse emittance blow-up factors as a function of the stripper thickness.

\begin{tabular}{|l|l|l|l|l|}
\hline Thick. & Rel. momen- & Stripp- & Meas'd B-up & Theor. B-up \\
\hline
\end{tabular} \begin{tabular}{|c|c|c|c|c|c|c|}
\hline [mm $]$ & tum loss & ing eff. & HOR & VERT & HOR & VERT \\
\hline 0.5 & & &
\end{tabular}

\begin{tabular}{|c|c|c|c|c|c|c|}
\hline 0.5 & $1.510^{-3}$ & $90 \%$ & 2 & 3.3 & 1.2 & 1.3 \\
\hline 1 & $3.010^{-3}$ & $95 \%$ & 2.3 & 4.2 & 1.5 & 2 \\
\hline 2 & $6.010^{-3}$ & $100 \%$ & 2.6 & 4.7 & 1.9 & 2.5 \\
\hline
\end{tabular}

Relative momentum losses and stripping efficiencies were in good agreement with theory [7, 8], but excessive emittance blow-ups (more than a factor two above the theoretical expectations) were measured. This seems to indicate a further optical problem, under investigation, in the transfer line.

\section{BEAM DIAGNOSTICS}

The six beam current transformers in the PSB injection line and the four DC ones in the rings were redesigned to cope (in PPM with high-intensity proton cycles) with the new low-intensity range. Pulse to pulse range switching is used in the injection line, auto-ranging in the rings. The new transformers use multi-layer shielding against external fields. The dynamic range of the PSB orbit measurement system has been extended to measure ion orbits. In the PS, the sensitivity of the wall current monitors has been increased.

Before and after the stripping foil in the PS-SPS beam line, two new identical transformers with a common calibrator help in the evaluation of the stripping efficiency.

\section{PERFORMANCES}

Overall performances are summarized in Table III.

Table III: Ion intensities at various stages and characteristics of the extracted $\mathrm{Pb}^{53+}$ beam

\begin{tabular}{|c|c|c|c|}
\hline Machine & Stage & Ions $\times 10^{8}$ & Design $\times 10^{8}$ \\
\hline Linac3 $\mathrm{Pb}^{53+}$ & after stripper & 10 & 9.25 \\
\hline \multirow{2}{*}{$\mathrm{PSB}$} & injected & 7.2 & \\
\cline { 2 - 4 } & accelerated & 3.0 & 2.22 \\
\hline \multirow{2}{*}{ PS } & injected & 2.8 & \\
\cline { 2 - 4 } & accelerated & 1.6 & 1.48 \\
\hline
\end{tabular}

\begin{tabular}{|c|c|}
\hline Extracted Beam after Strip & \\
\hline Number of bunches & 20 \\
\hline Intensity & $1.5 \times 10^{8}$ ions/pulse \\
\hline Bunch length & $11 \mathrm{~ns}$ \\
\hline Long. emittance & $0.037 \mathrm{eVs} / \mathrm{u}$ \\
\hline Momentum spread $(2 \sigma)$ & $0.4 \times 10^{-3}$ \\
\hline Emittance $(2 \sigma)$, hor., vert. & $1.5 \mu \mathrm{m}, 0.6 \mu \mathrm{m}$ \\
\hline
\end{tabular}

\section{CONCLUSIONS}

In the past the PS circular machines proved able to handle ions: deuterons and alphas in the seventies, oxygen and sulfur ions in the eighties. Lead ions, however, required a more substantial upgrading due to their much lower velocity and due to their sensitivity to the residual vacuum. Especially with the latter one is at the limit of what could be achieved at reasonable cost and within a short time. It can be noted that most of the predictions turned out to be fairly accurate, as shown by the successful acceleration of lead ions to more than design intensity in the CERN PS and its Booster.

\section{ACKNOWLEDGMENTS}

We would like to thank C. Carter, S. Hancock, J. P. Terrier, J. L. Vallet, and the PS and PSB operation teams, in particular B. Vandorpe, for their efficient support during preparation and operation for physics.

\section{REFERENCES}

[1] D. Warner ed., CERN Heavy Ion Facility Design Report CERN 93-01, April 1993.

[2] H. Haseroth, The CERN Heavy Ion Accelerating Facility, this Conference.

[3] B. Franzke, Vacuum Requirements for Heavy Ion Synchrotrons, IEEE Trans. Nucl. Sci. NS-28, 1981, p. 2116.

[4] M. van Rooij, J.-P. Bertuzzi, M. Brouet, A. Burlet, C. Burnside, R. Gavaggio, L. Petty, A. Poncet, The Vacuum Upgrade of the CERN PS and PS Booster, this Conference.

[5] F. Pedersen, N. Rasmussen, H. Schönauer, Fast Capture of Heavy Ions in the CERN PS Booster, Proc. 4th Europ. Part. Acc. Conf., London, June 1994, pp. 12251227.

[6] F. Blas, J. Boucheron, B.J. Evans, R. Garoby, G.C. Schneider, J.P. Terrier, J.L. Vallet, Digital Beam Controls for Synchrotrons and Storage Rings in the CERN PS Complex, ibid., pp. 1568-1570.

[7] U. Fano, Penetration of Protons, Alpha Particles and Mesons, Ann. Rev. Nucl. Sci. 13, (1963), p 1.

[8] P. Thieberger, H.E. Wegner, J. Alonso, H. Gould, C. Munger, R. Anholt, W.E. Meyerhof, Fully Stripped Heavy Ion Yields vs. Energy for Xe and $\mathrm{Au}$ Ions, IEEE Trans. on Nucl. Sci., Vol NS-32, No.5, October 1985, p. 1767. 